\title{
Editorial: Mobile Multimedia Communications
}

\author{
Zheng Yan ${ }^{1,2} \cdot$ Wei Wang ${ }^{3} \cdot$ Yonggang Wen ${ }^{4} \cdot$ Chonggang Wang ${ }^{5} \cdot$ Honggang Wang ${ }^{6}$
}

Published online: 6 May 2017

(C) Springer Science+Business Media New York 2017

\section{Editorial:}

Successfully deploying multimedia services and applications in mobile environments requires adopting an interdisciplinary approach where multimedia, networking and physical layer issues are addressed jointly. Content feature analysis and coding, media access control, multimedia flow and error control, cross-layer optimization, Quality of Experience (QoE), media cloud, media data processing as well as mobility management and security protocols are research challenges that need to be carefully examined when designing new mobile media architectures. We also need to put a great effort in designing applications that take into account the way the user perceives the overall quality of provided services.

Within this scope, this special issue is intended to provide a unique international forum for researchers from industry and academia, working on multimedia coding, security, mobile communications and networking fields, to study new technologies,

Zheng Yan

zyan@xidian.edu.cn

1 The State Key Lab of Integrated Services Networks, School of Cyber Engineering, Xidian University, No. 2 South Taibai Road,

710071 Xi'an, People's Republic of China

2 Department of Communications and Networking, Aalto University, Konemiehentie 2, Espoo P.O. Box 15400, Finland

3 Department of Computer Science, San Diego State University, 5500 Campanile Dr, San Diego, CA 92182, USA

4 School of Computer Science and Engineering (SCSE), Nanyang Technological University (NTU), 50 Nanyang Ave, Singapore 639798, Singapore

5 InterDigital Communications, Wilmington, DE, USA

6 University of Massachusetts (UMass) Dartmouth, 285 Old Westport Rd, North Dartmouth, MA 02747, USA applications and standards. Original unpublished contributions are solicited that can improve the knowledge and practice in the integrated design of efficient technologies and the relevant provision of advanced mobile multimedia applications.

This special issue features six selected papers with high quality. The first article, "A Survey of Verifiable Computation", authored by Yu et al., provided a thorough survey on the current state of art of verifiable computation in cloud computing to verify the correctness of data processing at the cloud in order to enhance its trustworthiness, especially for encrypted data processing. This survey gives a comprehensive review on this research field, compares the pros and cons of existing work based on performance evaluation metrics, highlights open research issues and indicates future research directions.

The second article titled "LTE-EMU: A High Fidelity LTE Cellar Network Testbed for Mobile Video Streaming" presented the design and implementation of an end-to-end network testbed for LTE cellar network called LTE-EMU in order to provide a convinced testbed for LTE cellar network. This testbed can reproduce the actual end-to-end transmission characteristics observed in real LTE networks by combining methods of both simulation and emulation.

In the next article with the title "Haze Removal via Edge Weighted Pixel-to-Patch Fusion", the authors proposed an efficient method for haze removal under the guidance of depth edges in order to accurately estimate the transmission map using strong priors or assumptions. In this method, a depth edge prior is applied to obtain the depth edges from a hazy image, and then a pixel-to-patch fusion scheme weighted by the depth edges is employed to estimate the transmission directly, which can preserve the sharp discontinuity at depth edges but smooth the surface texture in the rest regions of a transmission map.

In order to explore user readiness and adoption of sensing technologies to change user behaviors, the fourth article titled "EHR: A Sensing Technology Readiness Model for Lifestyle 
Changes" developed a model called EHR (e-health readiness) through user survey to investigate the relationship between user habits, perceived healthiness and beliefs towards sensing technologies, and how these factors influence user readiness to use sensing technologies to manage their wellness. The study result is helpful in designing future sensing technologies for behavior change.

For solving the problem that using static trust thresholds for misbehavior detection may result in high false positives, low malicious node detection rate, and network partitioning in Mobile Ad Hoc Networks (MANETs), the fifth article, "Isolating Misbehaving Nodes in MANETs with an Adaptive Trust Threshold Strategy" proposed an interesting Adaptive Trust Threshold (ATT) strategy and evaluated its performance with regard to robustness, energy efficiency, package delivery ratio and malicious node detection accuracy by comparing it with non-ATT schemes.

Device-to-Device (D2D) communication is a promising technology for the next generation mobile communication networks and wireless systems (5G). The last article titled "UAKA-D2D: Universal Authentication and Key Agreement Protocol in D2D Communications" proposed a universal authentication and key agreement protocol for securing D2D communications with the support on user roaming and inter-operator operations. By adopting Diffie-Hellman Key Exchange (DHKE) algorithm and employing message authentication code, the protocol achieves privacy-preserving session key generation and mutual authentication between D2D users.

Acknowledgements The guest editors would like to thank all reviewers for their efforts in reviewing manuscripts submitted to this special issue. We also thank the Edit-in-Chief, Dr. Imrich Chlamtac for his supportive guidance during the entire editing process. The special issue is sponsored by the National Key Research and Development Program of China (grant 2016YFB0800700), the NSFC (grants 61672410 and U1536202), the Project Supported by Natural Science Basic Research Plan in Shaanxi Province of China (Program No. 2016ZDJC-06), the 111 project (grants B08038 and B16037), and the Ph.D. Program Foundation of Ministry of Education of China (grant 20130203110006), as well as Aalto University.

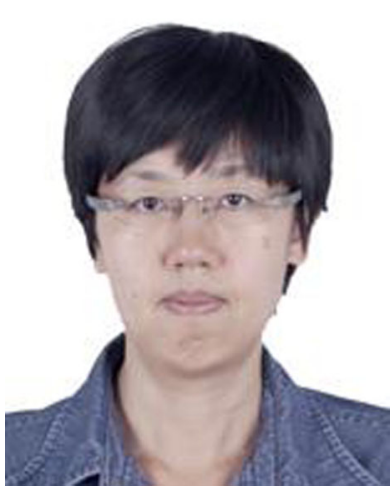

Zheng Yan (M'06 - SM'14) received her $\mathrm{PhD}$ degree at the Helsinki University of Technology. She is currently a Professor with Xidian University, Xi'an, China, and a Visiting Professor with Aalto University, Espoo, Finland. Her research interests include trust, security and privacy, social networking, cloud computing, networking systems, and data mining. Prof. Yan is an Associate Editor of Information Sciences, Information Fusion, the IEEE Internet of Things Journal, the IEEE Access Journal, JNCA, and Security and Communication Networks, etc. She is a leading Guest Editor of 20+ reputable journals, including ACM TOMM, Future Generation Computer Systems, the IEEE Systems Journal, JNCA, and MONET, etc. She is a Steering, Organization and Program Committee Member for more than 70 international conferences. She achieved IEEE Distinguished Leadership Award at IEEE TrustCom/BigDataSE/ISPA-2015; Outstanding Leadership Award at IEEE CIT 2014, CSS 2014, IEEE TrustCom 2013, and IEEE iThings 2012; Outstanding Service Award at IEEE TrustCom 2012; the Excellent Faculty of Xidian University in 2014; "100 Expert Plan" award of Shaanxi Province, China in 2011; and SisuAward, Nokia Research Center, Helsinki in 2010. She is a senior member of IEEE.

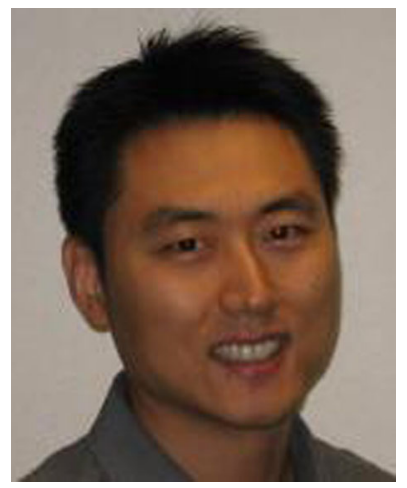

Wei Wang (M'10 - SM'16) received the Ph.D. degree in computer engineering from the University of Nebraska-Lincoln, USA, in 2009. He is an Assistant Professor with the department of Computer Science, San Diego State University, USA. He received his $\mathrm{Ph} . \mathrm{D}$. degree in Computer Engineering from University of Nebraska - Lincoln, USA, 2009. His major research interests include wireless multimedia communications, wireless sensor networks, network economics, and educational robotics. He won 2 Best Paper Awards of IEEE WCNC 2008 and ANSS 2011. He serves as an Associate Editor of Wiley Security in Communication Networks Journal, the Guest Editor of several Special Issues for various journals such as IEEE ACCESS and Springer MONET. He serves as the the web co-chair of IEEE INFOCOM 2016-2018, the program chair of ACM RACS 2014-2017, the workshop co-chair of ICST BodyNets 2013, the chair of IEEE CIT-MMC track 2012, the vice-chair of IEEE ICCT-NGN track 2011 and the program chair of the ICST IWMMN 2010, and a Technical Program Committee (TPC) member for many international conferences such as IEEE INFOCOM, GLOBECOM, and ICC.

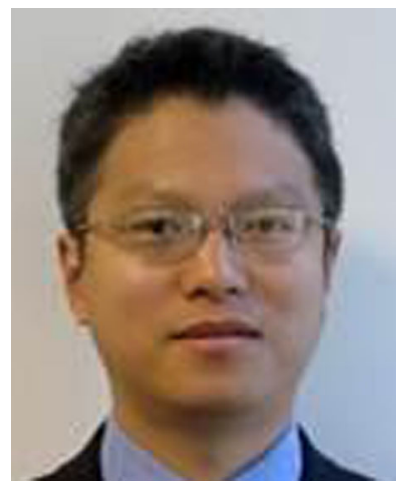

Yonggang Wen (S'99-M'08SM'14) is an associate professor with School of Computer Science and Engineering (SCSE) at Nanyang Technological University (NTU), Singapore. He received his $\mathrm{PhD}$ degree in Electrical Engineering and Computer Science (minor in Western Literature) from Massachusetts Institute of Technology (MIT), Cambridge, USA, in 2007. Previously he has worked in Cisco to lead product development in content delivery network, which had a revenue impact of 3 Billion US dollars globally. Dr. Wen has published over 150 papers in top journals and prestigious conferences. His work in Multi-Screen Cloud Social TV has been featured by global media (more than 1600 news articles from over 29 
countries) and received 2013 ASEAN ICT Awards (Gold Medal). His work on Cloud3DView, as the only academia entry, has won 2016 ASEAN ICT Awards (Gold Medal) and 2015 Datacentre Dynamics Awards 2015 - APAC ('Oscar' award of data centre industry). He is a co-recipient of 2015 I.E. Multimedia Best Paper Award, and a corecipient of Best Paper Awards at 2016 I.E. Globecom, 2016 I.E. Infocom MuSIC Workshop, 2015 EAI/ICST Chinacom, 2014 I.E. WCSP, 2013 I.E. Globecom and 2012 I.E. EUC. He received 2016 I.E. ComSoc MMTC Distinguished Leadership Award. He serves on editorial boards for IEEE Transactions on Circuits and Systems for Video Technology, IEEE Wireless Communication Magazine, IEEE Communications Survey \& Tutorials, IEEE Transactions on Multimedia, IEEE Transactions on Signal and Information Processing over Networks, IEEE Access Journal and Elsevier Ad Hoc Networks, and was elected as the Chair for IEEE ComSoc Multimedia Communication Technical Committee (2014-2016). His research interests include cloud computing, green data center, distributed machine learning, big data analytics, multimedia network and mobile computing.

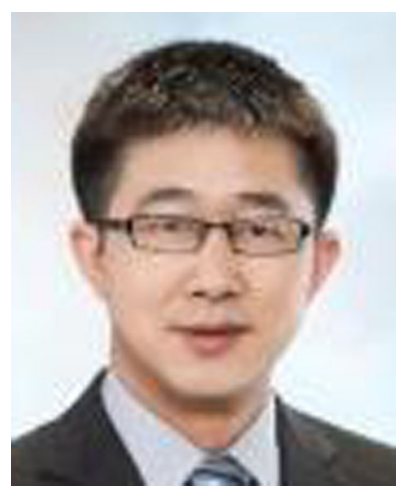

Chonggang Wang received his Ph.D. degree from Beijing University of Posts and Telecommunications (BUPT) in 2002. He is currently a Member Technical Staff with InterDigital Communications and leads a research theme on Internet of Things (IoT) and Big Data innovation. Prior to joining InterDigital in 2009, he had a multitude of R\&D experience in both academia and industry including NEC Laboratories America, AT\&T Labs Research, University of Arkansas-Fayetteville, and HongKong University of Science and Technology. He has abundant IoT standardization experience including rapporteur roles in and numerous contributions to one M2M, IETF, IEEE, and ETSI TC M2M. His current research interests include semantic computing and services for IoT, fog computing for IoT, IoT data analytics, and advanced IoT services. He has received several awards including long-term service award from IEEE ComSoc Multimedia Communications Technical Committee (2016), IEEE ComSoc Distinguished Lecturer (2015-2016), InterDigital's 2012 \& 2013 innovation award, outstanding leadership award from IEEE GLOBECOM 2010, and the national award for science and technology achievement in telecommunications in 2004 on Internet Protocol (IP) Quality of Service (QoS) from China Institute of Communications. He served as an NSF panelist on wireless networks in 2012 and was an external reviewer for Canada NSERC in 2013. He co-organized "Industry 4.0 vs. Internet of Things" session for the 2017 German-American Frontiers of Engineering (GAFOE) Symposium. His editorship experience within IEEE encompasses multiple roles. He is the co-founder and the founding Editor-inChief of IEEE Internet of Things Journal (2014-2016). He is currently an
Associate Editor-in-Chief of IEEE Transactions on Big Data and was on the editorial board of several journals including IEEE Wireless Communications Magazine, IEEE Communications Magazine, IEEE Transactions on Network and Service Management, and IEEE Access. $\mathrm{He}$ is also on the advisory board of IEEE-The Institute. His leadership background also includes the IEEE Computer Society Fellow Evaluation Committee Member (2017) and the chair of IoT Industry Community, IEEE Communications Society (2016-Present). Dr. Wang was elected an IEEE Fellow in 2017 for his contributions to IoT enabling technologies.

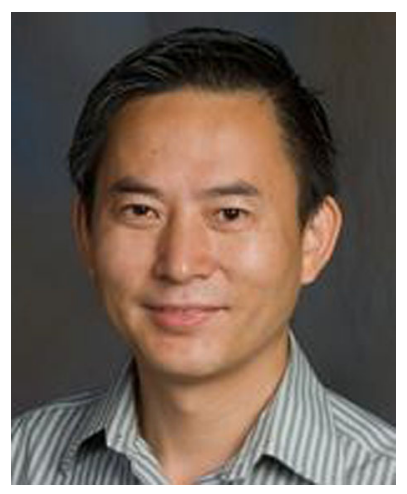

Honggang Wang received his Ph.D. in Computer Engineering at University of NebraskaLincoln in 2009. He is an associate professor at University of Massachusetts (UMass) Dartmouth. He is also the faculty member of Biomedical Engineering and Biotechnology Ph.D. program (BMEBT) at UMass Dartmouth. He has been a faculty member of Data Science master program of UMass Dartmouth since 2015. His research interests include Wireless Health, Body Area Networks (BAN), and BIG DATA in mHealth, Cyber and Multimedia Security, Mobile Multimedia and Cloud, Wireless Networks and Cyber-physical System. He serves as an Associate Editor of IEEE Transaction on Multimedia, an Associate Editor of IEEE transactions on Big Data, an Associate Editor-in-Chief of the IEEE Internet of Things Journal, an Associate Technical Editor of IEEE Communication Magazine, a Guest Editor of IEEE IoT Journal special issue on "IoT for Smart and Connected Health". He also serves as TPC Chair or Co-Chair for several conferences such as TPC Chair of 8th ICST/ACM International Conference on Body Area Networks (BODYNETS 2013), TPC symposium Co-Chair of IEEE conference on communications 2015 (ICC 2015, Mobile and Wireless Networking symposium), TPC Chair of IEEE HEALTHCOM 2015, and TPC cochair of IEEE ISCC 2015 and TPC track co-chair for the "Cognitive, Cellular and Mobile Networks (CCM)" of IEEE ICCCN 2014-2015. $\mathrm{He}$ also serves as the steering committee co-chair of IEEE CHASE (IEEE Conference on Connected Health: Applications, Systems and Engineering Technologies) and TPC co-chair of IEEE CHASE 2016, which is a leading international conference in the field of connected health He received IEEE Multimedia Communications Technical Committee (MMTC) Outstanding Leadership Award (2015), and IEEE HEALTHCOM 2015 Outstanding Service Award. He is the secretary of IEEE COMSOC e-Health Committee. His research is supported by DoT, UMass President Office, and UMass Healey Grant. His research is reported by media such as USA ABC 6 TV and Standard Times Newspaper. He received UMass Dartmouth Sponsored Research Recognition Award in 2015 and "Schorlar of The Year" award (only one per year) from UMass Dartmouth. 